\title{
APLICAÇÃO DO MODELO MONTE CARLO NA AVALIAÇÃO DA EMPRESA AMBEV COM CUSTO DE CAPITAL IMPRECISO
}

APPLICATION OF THE MONTE CARLO METHOD IN EVALUATION OF THE AMBEV COMPANY WITH UNUSUAL CAPITAL COST

\section{APLICACIÓN DEL MODELO MONTE CARLO EN LA EVALUACIÓN DE LA EMPRESA AMBEV CON COSTE DE CAPITAL IMPRECISO}

\section{Recebimento: 13/08/2018 - Aceite: 21/12/2018 - Publicação: 31/01/2019 \\ Processo de Avaliação: Double Blind Review}

Carla Vieira Silva ${ }^{1}$

Graduada em Admnistração

UFMG - Universidade Federal de Minas Gerais

carlavieira25@hotmail.com

\author{
Alexandre Teixeira Norberto Batista \\ Mestre em Desenvolvimento Econômico e Estratégia Empresarial \\ UFMG - Universidade Federal de Minas Gerais \\ alexandretnb@yahoo.com.br \\ Handerson Leonidas Sales \\ Mestre em Administração \\ UFMG - Universidade Federal de Minas Gerais \\ handerson.leonidas@hotmail.com \\ Roberto Silva da Penha \\ Mestre em Ciências Contábeis \\ UFMG - Universidade Federal de Minas Gerais \\ robertorrcontabilidade@hotmail.com
}

\section{RESUMO}

Uma das formas mais utilizadas para se apurar o valor de uma companhia (valuation) é o fluxo de caixa descontado, método que utiliza dados financeiros e contábeis da empresa para calcular o seu valor justo, com base na projeção de benefícios futuros de caixa. No entanto, apesar de ser o modelo mais utilizado, este método, pode não incorporar de maneira adequada os riscos do valuation, como o risco do custo de capital, que é impreciso em países emergentes, devido

\footnotetext{
${ }^{1}$ Autor para correspondência: Av. Pres. Antônio Carlos, 6627 - Pampulha, Belo Horizonte - MG, 31270-901
}

Revista ENIAC Pesquisa, Guarulhos (SP), V.8, n.1, jan.- jun. 2019. 
as constantes flutuações de taxas de juros, inflação e do próprio mercado. Uma forma de incorporar aos cálculos estes riscos, é usando distribuições de probabilidade com simulações Monte Carlo para se determinar previsões de diversos valores que a empresa pode assumir. Diante do exposto, o presente trabalho tem como principal objetivo verificar a acurácia da utilização da simulação de Monte Carlo no processo de avaliação de uma empresa através do método de fluxo de caixa descontado, incluindo a incerteza do cálculo de custo de capital. Após a análise dos resultados, chegou-se à conclusão de que a simulação de Monte Carlo é uma poderosa ferramenta para auxiliar na tomada de decisão, pois apesar de não prever o valor exato da empresa, ajuda a compreender os riscos e ameniza a subjetividade da avaliação, além de que permite conhecer os diversos valores que uma empresa pode assumir em diferentes cenários econômicos. $\mathrm{O}$ valor encontrado para a empresa no modelo determinístico está próximo da média das simulações, o mesmo ocorre com o custo de capital.

Palavras-chave: Valuation; Monte Carlo; Custo de Capital.

\section{ABSTRACT}

One of the most commonly used methods to determine a company's value is the discounted cash flow (DCF), a method that consider financial and accounting data to measure its fair value, based on the projection of future cash flow benefits. However, despite being the most used model, this method may not adequately incorporate valuation risks, such as the risk of capital cost, which is imprecise in emerging countries, due to the constant fluctuations of interest rates, inflation and the market itself. One way to incorporate such risks in the model is to use probability distributions with Monte Carlo simulations to determine predictions of various values that a firm can undertake. Regarding to this, the main objective of this work is to verify the accuracy of the use of Monte Carlo simulations in the process of valuation of a company through the discounted cash flow method, including the uncertainty of the cost of capital assumption. After the analysis, it was concluded that the Monte Carlo simulations is a powerful tool to support decision making, because although it does not predict the exact value of the company, it helps understanding the risks and softens the subjectivity of valuation, allowing to know a range of values that a company can assume in different economic scenarios. The value found for the firm in the deterministic model is close to the average of the simulations, as is the cost of capital.

Kewords: Valuation; Monte Carlo Method; Cost of Capital.

\section{RESUMEN}

Una de las formas más utilizadas para determinar el valor de una compañía (valuación) es el flujo de caja descontado, método que utiliza datos financieros y contables de la empresa para calcular su valor justo, sobre la base de la proyección de beneficios futuros de caja. Sin embargo, a pesar de ser el modelo más utilizado, este método, puede no incorporar de manera adecuada los riesgos de la valuación, como el riesgo del costo de capital, que es impreciso en países emergentes, debido a las constantes fluctuaciones de tipos de interés, inflación y del propio mercado. Una forma de incorporar a los cálculos estos riesgos, es usando distribuciones de probabilidad con simulaciones Monte Carlo para determinar predicciones de diversos valores que la empresa puede asumir. En el presente trabajo, el presente trabajo

Revista ENIAC Pesquisa, Guarulhos (SP), V.8, n.1, jan.- jun. 2019. 
tiene como principal objetivo verificar la exactitud de la utilización de la simulación de Monte Carlo en el proceso de evaluación de una empresa a través del método de flujo de caja descontado, incluyendo la incertidumbre del cálculo del costo de capital. Después del análisis de los resultados, se llegó a la conclusión de que la simulación de Monte Carlo es una poderosa herramienta para auxiliar en la toma de decisión, pues a pesar de no predecir el valor exacto de la empresa, ayuda a comprender los riesgos y ameniza la subjetividad de la empresa. evaluación, además de que permite conocer los diversos valores que una empresa puede asumir en diferentes escenarios económicos. El valor encontrado para la empresa en el modelo determinista está cerca de la media de las simulaciones, lo mismo ocurre con el costo de capital.

Palabras clave: Valuation; Monte Carlo; Costo de Capital.

\section{INTRODUÇÃO}

A gerência financeira é condição essencial para que a organização alcance seus objetivos, como a maximização do valor para seus acionistas (MEDEIROS NETO, 2009). Para isso, é fundamental conhecer os principais indicadores e metodologias utilizadas para a avaliação de uma empresa. Um desses indicadores é o Valuation, que visa identificar o valor justo de mercado de uma empresa, e faz uso de diversos dados financeiros e projeções para que se obtenha uma precificação precisa. Esse indicador permite conhecer aspectos interessantes da empresa, como, por exemplo, os fatores que impactam no seu valor de mercado e, adicionalmente, permite a um investidor tomar melhores decisões na hora de investir, pois com o Valuation, ele não baseará suas decisões em aspectos subjetivos evitando precificar um ativo com valor discrepante da sua realidade.

Essa avaliação é aplicável na decisão de captação de investimento, nos processos de fusão, cisão, Initial Public Offering (IPO), na realização da venda ou compra de uma empresa e qualquer outro tipo de negociação na qual a empresa esteja envolvida. Além disso, subsidiam estratégias de aperfeiçoamento da gestão empresarial. Atualmente, o modelo mais aceito para mensurar o Valuation de um ativo é o fluxo de caixa descontado Discounted Cash Flow (DCF) atendendo com um maior rigor à teoria de Finanças. Esse método possibilita estimar o valor presente de um ativo através de uma sequência de fluxos de caixa futuros descontados por uma taxa de juros (ASSAF NETO, 2010).

Contudo, é um método que gera algumas incertezas, por ser um modelo baseado em expectativa futura. Avaliar o valor de uma empresa é uma tarefa complexa, uma vez que se

Revista ENIAC Pesquisa, Guarulhos (SP), V.8, n.1, jan.- jun. 2019. 
baseia em resultados obtidos do comportamento do mercado, e estes são influenciados por diversos fatores, os quais não são controláveis no processo de projeção (ASSAF NETO, 2010). A maior fonte de incerteza durante o processo de avaliação de uma empresa pelo método de fluxo de caixa descontado é a estimativa dos fluxos de caixa futuros e a taxa de desconto que irá ser utilizada para trazer esses fluxos a valor presente (GARRAN, 2006).

Abordagens tradicionais de Valuation dentro de uma estrutura determinística são alvos de algumas críticas levantadas por Massari, Gianfrate e Zanetti (2016). Esses autores apontam que que as decisões gerenciais futuras de um investimento são tomadas no tempo em que o modelo de previsão é configurado, sem permitir revisões futuras do gerenciamento estratégico sobre a vida útil da empresa. Outra crítica corresponde ao uso de apenas uma única taxa de desconto, ou seja, são condições determinísticas sobre um futuro ou cenários ainda não realizados.

Além das críticas apontadas, Assaf Neto, Lima e Araújo (2006), mostram que há diversos problemas na utilização de algumas informações em países emergentes, devido à instabilidade de seus valores, causando um desequilíbrio dos indicadores financeiros e econômicos do país e impedindo uma definição de tendência que seja confiável. Segundo Garran (2006), é necessário que seja feita uma adaptação desses modelos para que eles possam se adequar à realidade do mercado. $\mathrm{O}$ autor ainda sugere a criação de um modelo próprio, que seja menos restritivo e que possa se adequar melhor à realidade macro e microeconômica brasileira, e ainda, que pudesse absorver diversos fatores de risco que existem em uma economia emergente, os quais devem ser levados em conta no processo de avaliação.

Uma forma de mensurar os riscos em um ambiente instável é aplicando modelos estocásticos na apuração do Valuation, já que eles incorporam a complexidade do ambiente em que as empresas estão inseridas, por meio de simulações de diversos cenários possíveis. Nesse sentido, a palavra "estocástica" pode ser entendida como sinônimo para randômica ou probabilística, e um antônimo para determinística.

Segundo, Medeiros Neto (2009) algumas pesquisas anteriores indicam o uso de ferramentas estocásticas como alternativa adicional para análise e mensuração de riscos. Ainda segundo o autor, esta é uma das principais contribuições desta ferramenta, já que o método tradicional (determinístico) não incorpora os riscos das variáveis como o modelo estocástico. Uma ferramenta útil na área financeira para analisar variáveis estocásticas em processos de

Revista ENIAC Pesquisa, Guarulhos (SP), V.8, n.1, jan.- jun. 2019. 
avaliação e análise de risco é o método de Monte Carlo, pois possibilita a simulação de cenários futuros a partir de distribuições de probabilidade para os parâmetros definidos (MEDEIROS NETO, 2009)

Ressalta-se que a prática de utilização de modelos estocásticos é ainda limitada, assim como os trabalhos em finanças que dedicam seções a metodologia que incorpora os riscos e que ameniza as falhas do modelo determinístico, como depreendem Damodaran (2014) e Bruni (2013). Menciona-se, ainda, que este trabalho tem maior preocupação com as variáveis que formam a taxa de desconto (WACC), considerando que esta irá assumir diversos valores no futuro (MASSARI; GIANFRATE; ZANETTI, 2016).

Diante do exposto, sob o efeito de variações do ambiente corporativo, principalmente do custo de capital, levanta-se a seguinte questão norteadora: Qual a acurácia da utilização da simulação de Monte Carlo no processo de avaliação de uma empresa por meio do método de fluxo de caixa descontado, incluindo a incerteza do cálculo de custo de capital?

O presente trabalho tem como objetivo verificar a acurácia da utilização da simulação de Monte Carlo no processo de avaliação de uma empresa através do método de fluxo de caixa descontado, incluindo a incerteza do cálculo de custo de capital. Contribui, portanto, para o processo de avaliação de empresas, na tomada de decisão em cenários e ambientes de incerteza, e com a literatura acerca da utilização desse método estatístico para o cálculo do Valuation, denominado por alguns de Monte Carlo Valuation (MASSARI; GIANFRATE; ZANETTI, 2016).

A empresa escolhida como objeto de análise para apuração do valor de mercado e simulações de probabilidade foi a Ambev S/A. A escolha da companhia é justificada por se tratar de um "case de sucesso" no Brasil como uma das empresas que mais obteve retorno sobre o valor das suas ações na última década. Segundo a consultoria Economatica ${ }^{\circledR}$ (2018), a Ambev foi a empresa brasileira líder em valor de mercado, desde dezembro de 2014 até abril de 2018, se mantendo constantemente nessa colocação. Em maio desse mesmo ano, a empresa perdeu a colocação para a Petrobras.

Revista ENIAC Pesquisa, Guarulhos (SP), V.8, n.1, jan.- jun. 2019. 


\section{REVISÃO DA LITERATURA}

\subsection{AVALIAÇÃO DE EMPRESAS E ESTRUTURA DOS MODELOS TRADICIONAIS}

O valor de uma empresa é formado por quatro fatores, que são: "sua capacidade de gerar fluxos de caixa a partir dos ativos já instalados, a taxa de crescimento esperada desse fluxo de caixa, o tempo transcorrido até a empresa alcançar o crescimento estável e o custo de capital" (DAMODARAN, 2004 p. 611). Sendo assim, a avaliação de empresas deve considerar algumas premissas que correspondem ao contexto e mercado em que a organização está inserida, às hipóteses comportamentais e às expectativas de resultado futuro. A confiabilidade e a qualidade dos dados financeiros são indispensáveis para que se possa encontrar um valor justo e preciso para a organização.

Nesse contexto da busca do justo valor, o método do valor de uma empresa não se baseia em uma ciência exata, a qual é possível comprovar e ter certeza absoluta de seus resultados. Os fatores que são analisados, buscam representar a realidade e o contexto do ambiente econômico onde a empresa se encontra, e estes, são representados em hipóteses ou premissas, por exemplo, todos os modelos de avaliação desenvolvem expectativas para a projeção dos resultados futuros. Sendo assim, mesmo buscando quantificar esses valores da melhor forma possível, é comum que a avaliação tenha uma margem de arbitro do analista. Para um cálculo de um valor justo de uma empresa é necessário que seja adotado um método coerente e que tenha premissas não viesadas em fundamentos de negócios (ASSAF NETO, 2010).

De acordo com Damodaran (1997) existem três principais abordagens para apurar o Valuation. A primeira é a avaliação por fluxo de caixa descontado, para essa abordagem, onde o valor de um ativo é o valor de seus fluxos de caixa futuros trazido a valor presente. A segunda é a avaliação relativa, que busca estimar o valor de um ativo focando a precificação de ativos semelhantes e que sejam comparáveis em variáveis em comum, como por exemplo, o lucro; a terceira, é a avaliação de direitos contingentes.

Os modelos tradicionais de fluxo de caixa descontado, dentre todos, os mais utilizados, apresentam uma fragilidade em seu resultado por não levarem em conta de forma adequada a variabilidade e complexidade do mundo, os cálculos e análises podem resultar em avaliações falhas. Além de que, presume-se que todas as decisões sobre a gestão futura da empresa ou de um investimento, são feitas no momento em que o modelo de avaliação está sendo criado. As

Revista ENIAC Pesquisa, Guarulhos (SP), V.8, n.1, jan.- jun. 2019. 
avaliações tradicionais de fluxo de caixa se baseiam no pressuposto de que os fluxos de caixa futuros são previsíveis e determinísticos, e utilizam taxas ambíguas que em muitos casos não permitem uma avaliação clara dos riscos inerentes a um investimento (MASSARI; GIANFRATE; ZANETTI, 2016).

De acordo com Assaf Neto (2010) a forma como o custo de capital é calculado no Brasil possui algumas limitações e podem trazer perda da qualidade do resultado apurado. O método que é usado para calcular o custo de capital próprio Capital Asset Pricing Model (CAPM), foi feito para ser utilizado em economias estáveis. Portanto, métodos mais sofisticados e adaptados devem ser escolhidos e aplicados para o mercado emergente, como é o caso do Brasil.

É evidente que o uso de algumas variáveis como a taxa livre de risco, que é utilizada para o cálculo do custo de capital da empresa, possuem pouca validade em avaliações feitas no Brasil, já que não é possível obter uma média histórica confiável, o que torna questionável o método que é utilizado na avaliação. Ainda de acordo com os autores, é possível apontar a instabilidade do valor da taxa SELIC como um dos motivos da discrepância dos valores que são encontrados nos desvio-padrão e coeficiente de variação dos períodos que foram analisados durante o estudo. Já no cálculo do prêmio do risco de mercado, segundo os autores, há problemas no que se refere à qualidade e volatilidade das informações, prejudicando definições de tendências que sejam confiáveis (ARAUJO; LIMA; ASSAF NETO, 2006).

Para mitigar as várias restrições na apuração do valor de mercado de uma empresa, algumas delas apresentadas nesta seção, modelos estocásticos envolvendo distribuições de probabilidade das variáveis que influenciam esse valor são uma opção.

\subsection{O MÉTODO MONTE CARLO E SEU USO EM FINANÇAS}

De acordo com Cardoso e Amaral (2000, p. 5), o método de Monte Carlo é uma "tradicional técnica que usa número aleatórios e pseudo-aleatórios para retirar amostras de uma distribuição de probabilidades". Ainda segundo os autores, o termo Monte Carlo surgiu durante a Segunda Guerra Mundial, e era utilizado como um código para problemas de simulação durante o desenvolvimento de uma bomba atômica.

Conforme, Cardoso e Amaral (2000, p. 4) essa ferramenta auxilia a qualidade das análises, pois “sem a ajuda da simulação, uma planilha revela apenas uma simples saída, ou a

Revista ENIAC Pesquisa, Guarulhos (SP), V.8, n.1, jan.- jun. 2019. 
mais provável, ou um cenário médio. Esta é a grande causa de divergências entre valores orçados (ou previstos) e os reais, quando certas variáveis ambientais não são consideradas".

Uma vez entendidos algumas das premissas e dos principais conceitos que envolvem a simulação de Monte Carlo, faz-se necessário entender o processo de simulação, no sentido de que cada variável que foi determinante na construção do modelo tradicional DCF, será considerada como uma premissa, ou uma variável aleatória, definida em uma função densidade de probabilidade, que permitirá obter uma amplitude de resultados, por meio de várias iterações, como ilustrada na Figura 1, baseada no modelo de French e Gabrielli (2005).

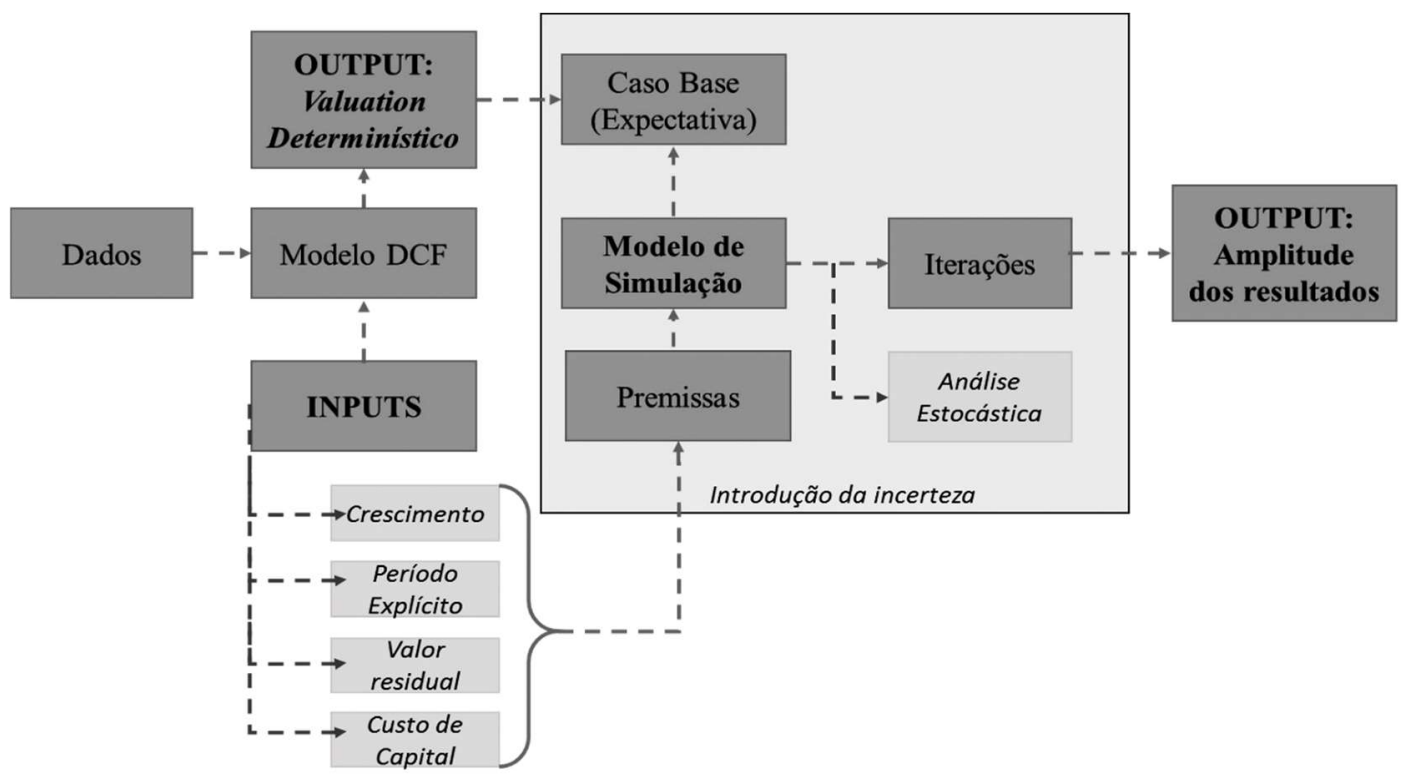

Figura 1: O processo de simulação

Fonte: Adaptado de French e Gabrielli (2005)

Correia Neto, Moura e Forte (2002) salientam a importância da estatística na avaliação de uma empresa. De acordo com os autores, o método de Monte Carlo gera de forma contínua e aleatória diversos cenários possíveis de ocorrerem, tal geração randômica garante que os resultados não sejam influenciados por uma inclinação pessimista ou otimista daquele que está realizando as projeções. Cada simulação corresponde a um cenário que é possível ocorrer e consequentemente todas as simulações são guardadas em uma distribuição de probabilidade. A forma como esses eventos estão dispostos possibilita a avaliação da probabilidade de cada cenário ocorrer, por meio de estatística descritiva.

Revista ENIAC Pesquisa, Guarulhos (SP), V.8, n.1, jan.- jun. 2019. 


\subsection{ESTUDOS ANTERIORES}

Oliveira et al (2017) realizaram um estudo que mostrou de forma prática algumas reflexões que podem ajudar na utilização de algumas variáveis contábeis para a apuração do Valuation. A empresa escolhida foi a Petrobras e para encontrar o valor dessa empresa foram levantados seus dados financeiros e os dados do setor no qual ela estava inserida. Após os cálculos tradicionais do fluxo de caixa descontado utilizados pelos autores, foi encontrado um valor negativo para a ação da referida empresa, mesmo esta possuindo ações que compõem o índice IBOVESPA o que demonstra uma elevada negociação de suas ações no mercado de capitais. Isso mostra a importância de não se deixar levar por tendências do mercado e saber utilizar as ferramentas e técnicas corretas para se fazer uma avaliação correta e não fazer um mal negócio.

Santos e Cunha (2015) elaboraram um estudo com o objetivo de identificar se a prestadora de serviço que realiza a avaliação de uma empresa pode causar um viés do preço da ação. Os autores analisaram 106 laudos que foram publicados entre 2002 e 2013, através de um método estatístico e concluíram que existe um grande viés na avaliação, especialmente quando a contratante da avaliação é a própria empresa avaliada.

Schnorrenberger et al (2015) concluíram em seu estudo que o método escolhido pelo analista pode interferir no valor final que a empresa pode assumir, mostraram que há uma grande variação do valor encontrado pelo método de fluxo de caixa descontado e pelo método contábil. Tal estudo mostra a importância de se discutirem as falhas e propor melhorias das metodologias que são utilizadas para apurar o Valuation.

De acordo com qualquer ato de projeção engloba riscos, que são, basicamente, o risco de não acontecer aquilo que foi projetado e os resultados previstos não se realizarem. Os autores fazem um estudo a fim de abordar a utilização da estatística como forma de tentar amenizar a incerteza dos riscos na avaliação de uma empresa. A conclusão do estudo foi de que o método estocástico se mostrou adequado no processo de avaliação da empresa, pois além de criar diversos cenários futuros de forma aleatória, possibilitou a quantificação do risco da empresa (CORREIA NETO; MOURA; FORTE, 2002)

Tibor, Veronika e Réka (2010) realizaram um estudo que utilizou três métodos de avaliação de empresas para tentar obter resultados mais precisos. Os métodos combinados

\footnotetext{
Revista ENIAC Pesquisa, Guarulhos (SP), V.8, n.1, jan.- jun. 2019
} 
foram o fluxo de caixa descontado, a avaliação relativa e a avaliação contingente. Para obter uma estimativa de valor mais eficiente e tentar amenizar os riscos, utilizaram o método de Monte Carlo. Os autores chegaram a um valor de mercado próximo ao valor do mercado atual, o que demonstra a viabilidade de se testar novos métodos de cálculo do Valuation.

Amsler e Schmidit, em 1985 tentaram identificar a precisão de alguns testes estatísticos através do método de Monte Carlo, e chegaram à conclusão de que alguns testes que eram usados para o cálculo do CAPM não eram confiáveis, mesmo que a quantidade de dados fosse extremamente grande. Já o estudo de French e Gabrielli (2005) teve como objetivo identificar formas de incorporar ao método de fluxo de caixa descontado as incertezas na hora da avaliação, e para isso os autores utilizaram o programa Crystal Ball. O estudo conseguiu verificar que o valor que foi encontrado estava muito próximo ao valor de mercado, contudo, agora, o investidor teria mais compreensão das vantagens e desvantagens de uma avaliação e teria consciência dos valores que a empresa poderia assumir.

Os estudos citados mostram que os métodos utilizados atualmente para fazer uma avaliação de uma empresa possuem falhas, especialmente no que diz respeito à projeção e incorporação de alguns elementos que envolvem incertezas, como é o cálculo do custo de capital. Contudo, os estudos apresentados mostram que a incorporação da estatística na avaliação vem mostrando resultados positivos no que diz respeito à correção das falhas.

\section{METODOLOGIA}

\subsection{CLASSIFICAÇÃO DA PESQUISA}

Diante dos objetivos mencionados anteriormente, o presente estudo tem caráter descritivo e exploratório. De acordo com Gil (2002), as pesquisas descritivas têm como principal objetivo a descrição de características de determinado fenômeno ou população, ou estabelecimento de relações entre variáveis. Ainda segundo o autor, uma das principais particularidades desse tipo de pesquisa, é a utilização padronizada de coleta de dados. A pesquisa possui uma abordagem qualiquantitativa, pois busca realizar uma análise estatística para testar as hipóteses levantadas, e envolve subjetividade no momento da avaliação.

Revista ENIAC Pesquisa, Guarulhos (SP), V.8, n.1, jan.- jun. 2019. 


\subsection{ORGANIZAÇÃO, COLETA E FILTRAGEM DOS DADOS}

Baseado no modelo de avaliação de empresas através do fluxo de caixa descontado, que é citado por diversos autores (MULLER; TELÓ, 2003; SOUTE et al, 2008) como o método mais completo para a avaliação de uma empresa, o presente estudo buscou realizar uma análise pelo modelo estocástico de Valuation, que contemple variações no valor de uma empresa oriundas da incerteza do cálculo de custo de capital.

Foi realizada uma análise determinística da empresa, para isso, utilizando-se de demonstrativos e indicadores financeiros da empresa AMBEV durante o período de 2013 a 2017. Com base no banco de dados Economatica ${ }^{\circledR}$ para organização, coleta e filtragem dos dados da empresa, os dados foram ajustados pelo IPCA para 31-12-2017.

Além dessas, outras variáveis foram necessárias para a apuração do valor da empresa. Os valores do Default Spread (risco de inadimplência) da empresa, que de acordo com o site da mesma, está classificado como Baa3 segundo a Moody's. A taxa equivalente a essa classificação é fornecida por Damodaran (2018c). O Valuation foi apurado pelo método de fluxo de caixa descontado, usando o modelo determinístico, e o modelo estocástico com simulações de probabilidade com auxílio de planilha eletrônica. O desenvolvimento do modelo estocástico de Valuation foi feito através do método de Monte Carlo.

\subsection{PREMISSAS DO MODELO DETERMINÍSTICO}

O primeiro passo para realizar o modelo determinístico foi analisar os dados financeiros da empresa. Foram selecionados os dados do período de 2013 a 2017, esse horizonte de tempo foi determinado por ser um período mais recente da empresa que está sendo analisada. Para se definir o custo de capital por meio do modelo de ponderação de capital, Weighted Average Capital Cost (WACC), foi calculado o retorno mínimo exigido sobre o capital próprio utilizando o modelo CAPM. Foram retirados do software Economatica ${ }^{\circledR}$ dados de fechamento e retorno da S\&P 500 e da empresa Ambev a fim de fossem calculados o Beta ( $\beta$ ), a Risk Free Rate $\left(R_{f}\right)$ e o Retorno de mercado $\left(R_{m}\right)$. Já o Spread da empresa, utilizou-se um rating classificado em Baa3 na Moody’s (AMBEV, 2018). Para apuração do custo da dívida foram utilizados a Risk Free Rate, Default Srepad e o Risco do País, segundo a Moody's

Revista ENIAC Pesquisa, Guarulhos (SP), V.8, n.1, jan.- jun. 2019. 
(DAMODARAN, 2018c). Os dados utilizados para o cálculo do custo de capital da empresa, estão expostos na Tabela 1.

Tabela 1: Dados utilizados para o cálculo do WACC

\begin{tabular}{c|l|l}
\hline Variável & \multicolumn{1}{|c}{ Descrição } & \multicolumn{1}{c}{ Fonte } \\
\hline$R_{f}$ & t- bonds dos últimos 10 anos & Damodaran (2018a) \\
\hline$\beta$ & Beta alavancado & Economatica ${ }^{\circledR}$ \\
\hline$R_{m}-R_{f}$ & Prêmio pelo risco incorrido & Damodaran (2018a) \\
\hline Default Spread & Rating AMBEV: Baa3 (Moody's) & Damodaran (2018b); AMBEV (2018) \\
\hline riscoBR & Rating do Brasil: Ba2 (Moody's) & Damodaran (2018c) \\
\hline$\% D$ & Estrutura de Capital & Economatica ${ }^{\circledR}$ \\
\hline
\end{tabular}

Fonte: Elaborado pelos autores.

O PIB de 2,69\% utilizado para o cálculo dos fluxos de caixa em perpetuidade foi baseado na mediana de projeção de crescimento do boletim Focus do Banco Central divulgado em 05 de janeiro de 2018 (FOCUS, 2018). As projeções realizadas foram feitas com base nas médias dos desempenhos operacionais da empresa.

\subsection{PREMISSAS DO MODELO ESTOCÁSTICO}

Durante a construção do modelo de simulação deste trabalho, foram adotados o custo de capital e o valor de mercado da empresa como variáveis de saída da simulação de Monte Carlo. Com base nas premissas do modelo determinístico, foram utilizados os mesmos dados e as mesmas fontes. O próximo passo foi definir as variáveis de entrada que seriam simuladas e quais premissas seriam utilizadas no processo de simulação. As distribuições foram escolhidas de forma que se ajustassem da melhor forma possível aos dados que estavam disponíveis.

- $\quad R_{m}$ - Para a simulação desta variável, foi utilizada a média de $10,26 \%$, com base nos últimos 10 anos dos retornos S\&P 500 (DAMODARAN, 2018a). O desvio padrão utilizado na simulação foi de $2 \%$. A frequência de distribuição utilizada foi a normal.

- $\quad R_{f}$ - Da mesma forma que o $R_{m}$, foi calculada a média dos últimos 10 anos com base nos títulos do tesouro Americanos t-bonds, que é de 4,29\% (DAMODARAN, 2018a). Já o desvio padrão utilizado foi de $1 \%$ e a frequência de distribuição foi a normal.

Revista ENIAC Pesquisa, Guarulhos (SP), V.8, n.1, jan.- jun. 2019. 
- $\quad \beta$ - Para a simulação dessa variável, foram extraídos do software Economatica ${ }^{\circledR}$ os valores dos betas dos últimos 60 meses ( 5 anos). A média encontrada foi de 0,51 e o desvio padrão utilizado na simulação foi de 0,02 . A simulação foi feita com uma distribuição normal.

- Default Spread - Para que fosse feita a simulação desta variável, foi utilizada uma distribuição triangular, que é uma distribuição de probabilidade que possui um valor mínimo, máximo e um valor mais provável de acontecer. Como valor mais provável, foi utilizado o rating atual da empresa de 1,98\%, como valor mínimo foi utilizado o valor 1,27\% (Baa2) e como valor máximo 2,38\% (Ba1), ambos obtidos nos relatórios de Damodaran (2018b).

- RiscoBR - A simulação dessa variável foi feita com a mesma lógica utilizada para simular o Spread, foi empregada uma distribuição triangular, sendo que, como mínimo foi utilizado o rating Ba1, de 2,88\%; como máximo, o rating Ba3, de $4,15 \%$ e a moda o rating atual do país, Ba2 de 3,46\% (DAMODARAN, 2018c).

- $\quad$ PIB - Para a simulação do PIB, foi utilizada uma distribuição triangular com os seguintes valores: 2,69\% baseado na mediana de previsão do boletim Focus do Banco Central, e como valor mínimo e máximo, foram utilizados respectivamente os valores de $0 \%$ e $3 \%$, Valores acima de 3\% estariam demasiadamente otimistas considerando a atual conjuntura econômica Brasileira.

- Estrutura de Capital (\%D) - Para simular a estrutura de capital da empresa, foram extraídos do software Economatica ${ }^{\circledR}$ dados dos últimos 17 trimestres da empresa, esse período de tempo reflete mais proximamente a estrutura alvo da empresa na contemporaneidade, já que utilizar um tempo mais longo poderia trazer aos cálculos informações que não condizem com a realidade atual da companhia. A distribuição utilizada foi a normal, com a média de $6,33 \%$ e desvio padrão de $1,58 \%$.

\section{ANÁLISE DOS RESULTADOS}

Para que fosse feita a avaliação da empresa pelo método de fluxo de caixa descontado, seguiu-se o processo de cinco etapas, sendo elas: 1) a análise do desempenho histórico, 2) estimativa do custo médio ponderado de capital, 3) a projeção dos fluxos de caixa futuros, 4)

Revista ENIAC Pesquisa, Guarulhos (SP), V.8, n.1, jan.- jun. 2019. 
estimativa do valor residual, e por último, 5) trazer os fluxos de caixa a valor atual. As etapas são expostas nas seções seguintes.

\subsection{CASO BASE}

Na Tabela 2, estão expostos os valores utilizados para o cálculo do WACC da empresa Ambev.

Tabela 2: Apuração do WACC para o caso base

\begin{tabular}{|c|c|c|}
\hline Componentes & Capital Próprio & Capital de Terceiros \\
\hline$R_{f}$ EUA & $4,29 \%$ & $4,29 \%$ \\
\hline Beta $\beta^{*}$ & 0,728 & - \\
\hline$R_{m}-R_{f}$ & $5,97 \%$ & - \\
\hline RiscoBR & $3,46 \%$ & $3,46 \%$ \\
\hline Spread Ambev & - & $1,98 \%$ \\
\hline$I R$ & - & $34 \%$ \\
\hline $\mathrm{Ke}$ & $12,10 \%$ & - \\
\hline$K d$ & - & $6,42 \%$ \\
\hline$\% \mathrm{D}^{*}$ & & $5,26 \%$ \\
\hline WACC & & $11,80 \%$ \\
\hline
\end{tabular}

*Regressão dos retornos diários ABEV3 e retornos S\&P500, no período de 03/01/2013 a 28/12/2017.

*Informações da empresa referem-se às publicações de 31/12/2017.

Fonte: Elaboração própria com dados da pesquisa.

Após os cálculos, foi encontrado um valor de custo de capital de 11,80\% para a empresa. Após a taxa ser apurada, seguiu-se com as projeções dos fluxos de caixa operacionais disponíveis. Foram analisados os demonstrativos e indicadores financeiros da empresa no período de 2013 a 2017, esta etapa é importante pois ajuda a compreender o comportamento de alguns indicadores da empresa no período de tempo que está sendo avaliado, com base nisso foram feitas as médias de crescimento conforme Tabela 3.

Revista ENIAC Pesquisa, Guarulhos (SP), V.8, n.1, jan.- jun. 2019. 
Tabela 3: Médias de reinvestimento e crescimento do NOPAT da empresa Ambev

$\begin{array}{llllll}\text { ANO } & 2013 & 2014 & 2015 & 2016 & 2017\end{array}$

$\begin{array}{llllll}\text { EBIT } & 19.995 & 19.364 & 20.741 & 17.767 & 16.575\end{array}$

Capital Investido

13.197

12.780

13.689

11.726

10.940

ROIC

47.347

51.608

51.194

52.471

51.042

Capex

$28 \%$

$25 \%$

$27 \%$

$22 \%$

$21 \%$

Depreciação

$-5.129$

$-5.306$

$-5.732$

$-6.089$

$-3.044$

2.721

2.924

3.395

3.649

3.645

$\Delta$ Capital de giro

621

$-92$

198

$-1.812$

139

$-1.787$

$-2.473$

$-2.139$

$-4.252$

739

b NOPAT

$14 \%$

$19 \%$

$16 \%$

$36 \%$

$-7 \%$

g NOPAT

$4 \%$

$5 \%$

$4 \%$

$8 \%$

$-2 \%$

$3,98 \%$

Média b

$15,60 \%$

Fonte: Economatica ${ }^{\circledR}$ com Valores em milhões de Reais, ajustados pelo IPCA.

A média do crescimento ( $\mathrm{g}$ ) do NOPAT da empresa nos últimos 5 anos, foi de 3,98\%, com base nesses dados, foram projetados os próximos 5 anos. Já os fluxos de caixa disponíveis da empresa (FCDE) foram apurados a partir da média da taxa de reinvestimento (b) do NOPAT dos últimos 5 anos. Os fluxos podem ser apurados deduzido a do Lucro Operacional parcela que, em média, é reinvestida na empresa, de 15,6\%. Com base nesses valores, foram projetados os fluxos operacionais da empresa disponíveis, que depois foram trazidos a valor presente. Para o cálculo do valor residual da empresa, foi estabelecido que após o $5^{\circ}$ ano, os fluxos de caixa cresceriam em perpetuidade acompanhando o crescimento da economia. A Tabela 4 apresenta as projeções do fluxo de caixa.

Tabela 4: Projeções do fluxo de caixa

\begin{tabular}{ccccccc}
\hline Projeções & $\mathbf{2 0 1 8}$ & $\mathbf{2 0 1 9}$ & $\mathbf{2 0 2 0}$ & $\mathbf{2 0 2 1}$ & $\mathbf{2 0 2 2}$ & P. continuo \\
\hline NOPAT & 12.950 & 13.452 & 13.974 & 14.517 & 15.080 & 15.665 \\
\hline (-) Reinvestimento & 2.021 & 2.099 & 2.180 & 2.266 & 2.353 & 2.444 \\
\hline FCDE & 10.929 & 11.353 & 11.794 & 12.251 & 12.727 & 13.221 \\
\hline
\end{tabular}

Fonte: Dados da pesquisa

Revista ENIAC Pesquisa, Guarulhos (SP), V.8, n.1, jan.- jun. 2019. 
O valor encontrado dos fluxos de caixa futuros trazidos a valor presente foi de $\mathrm{R} \$ 42,43$ bilhões, e o valor residual, de R $\$ 145,14$ bilhões, que trazido a valor presente ficou de $\mathrm{R} \$ 83,098$ bilhões, conforme Tabela 5, formando assim o valor de mercado da empresa.

Tabela 5: Valuation

\begin{tabular}{lc}
\hline a. Valor Explícito & 42.428 \\
\hline b. Valor Contínuo & 145.138 \\
\hline c. VP do Valor Contínuo & 83.098 \\
\hline d. $(\mathbf{a}+\mathbf{c})$ Valor da empresa & $\mathbf{1 2 5 . 5 2 6}$ \\
\hline
\end{tabular}

Fonte: Dados da Pesquisa.

Com base nos cálculos expostos, foi encontrado o valor de $\mathrm{R} \$ 125,5$ bilhões para a empresa AMBEV no início de 2018. Depois de encontrar o valor da empresa Ambev pelo método de fluxo de caixa descontado para que servisse de caso base para a simulação Monte Carlo, foi colocado em prática o principal objetivo do presente trabalho, apurar o Valuation da empresa para verificar se o resultado encontrado será um bom representante dentro de diversas possibilidades de valor, considerando as variações no custo de capital e no crescimento da economia.

\subsection{IMPLEMENTAÇÃO DO MÉTODO MONTE CARLO}

Após determinar os parâmetros que foram utilizados na simulação, foram realizadas 10.000 simulações, e de acordo com French e Gabrielli (2005) e Oliveira e Medeiros Neto (2012), esse número de simulações é suficiente para garantir que os resultados se estabilizem. Após a simulação do WACC, obtém-se os seguintes resultados, conforme Tabela 6 .

Tabela 6: Dados estatísticos da simulação do WACC

\begin{tabular}{lrlr}
\hline \multicolumn{3}{c}{ Valores de previsão } \\
\hline Avaliações & 10.000 & Curtose & 3,04 \\
Caso Base & $11,80 \%$ & Coeficiente de Variação & 0,1066 \\
Média & $10,55 \%$ & Mínimo & $6,05 \%$ \\
Mediana & $10,52 \%$ & Máximo & $14,46 \%$ \\
Desvio Padrão & $1,12 \%$ & Largura do Intervalo & $8,40 \%$ \\
Variância & $0,01 \%$ & Erro Padrão Média & $0,01 \%$ \\
Obliquidade & 0,0189 & & \\
\hline
\end{tabular}


Fonte: Dados da Pesquisa

De acordo com a Tabela 6, o valor médio obtido para o WACC após as simulações foi de $10,55 \%$, valor que está $10,59 \%$ abaixo do valor encontrado durante o cálculo determinístico, que foi de 11,80\%. É possível afirmar que dentre todas as possibilidades que foram simuladas o valor mínimo, ou seja, no melhor cenário possível de custo de capital valor que o WACC pode assumir é de $6,05 \%$, e o máximo, de $14,46 \%$. Através do desvio padrão é possível analisar o risco da variável e na simulação o desvio padrão obtido foi de $1,12 \%$. Nesse caso, valores do custo de capital entre $9,43 \%$ a $11,67 \%$ representaria a abrangência apresentada de frequência de ocorrência, ainda assim abaixo dos 11,8\% utilizado no cálculo determinístico. A distribuição dos valores encontrados na simulação pode ser verificada na Figura 2.

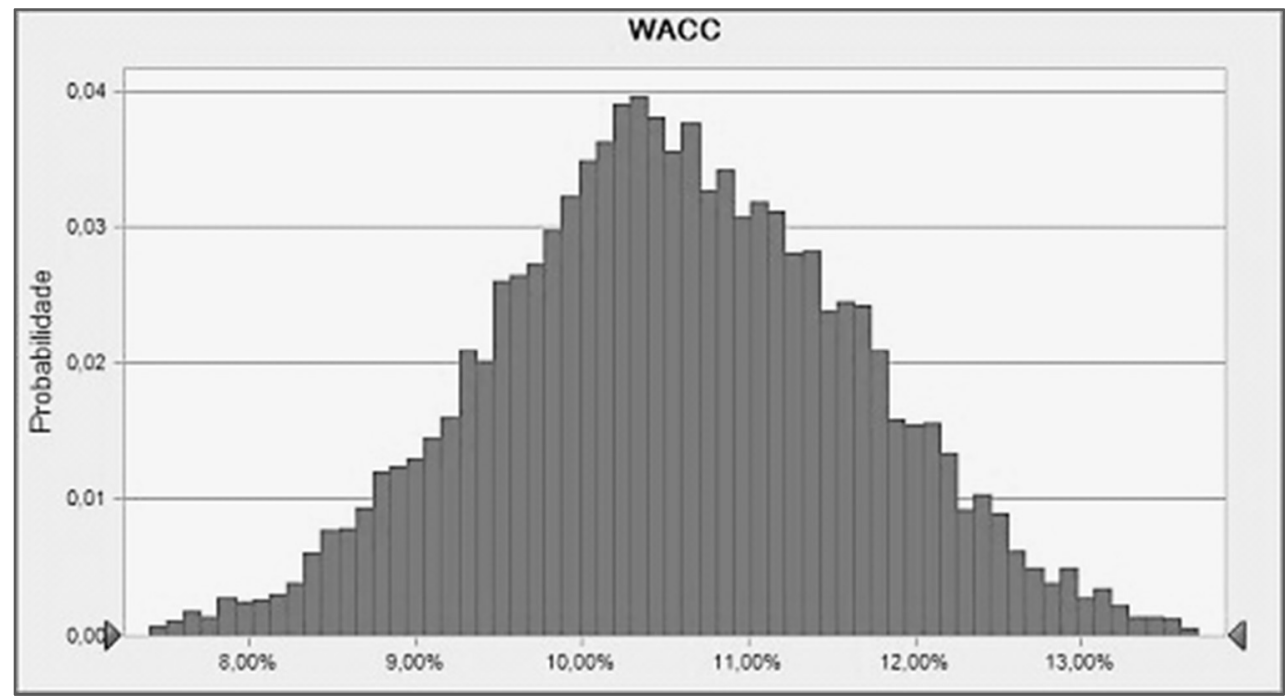

Figura 2: Distribuição de probabilidade do WACC Fonte: Dados da pesquisa.

Também foram analisadas as correlações geradas no momento da simulação, elas mostram quais variáveis afetam o resultado final do cálculo com maior intensidade, em outras palavras, quais dados contribuem mais para a variância da variável de saída.

Revista ENIAC Pesquisa, Guarulhos (SP), V.8, n.1, jan.- jun. 2019. 


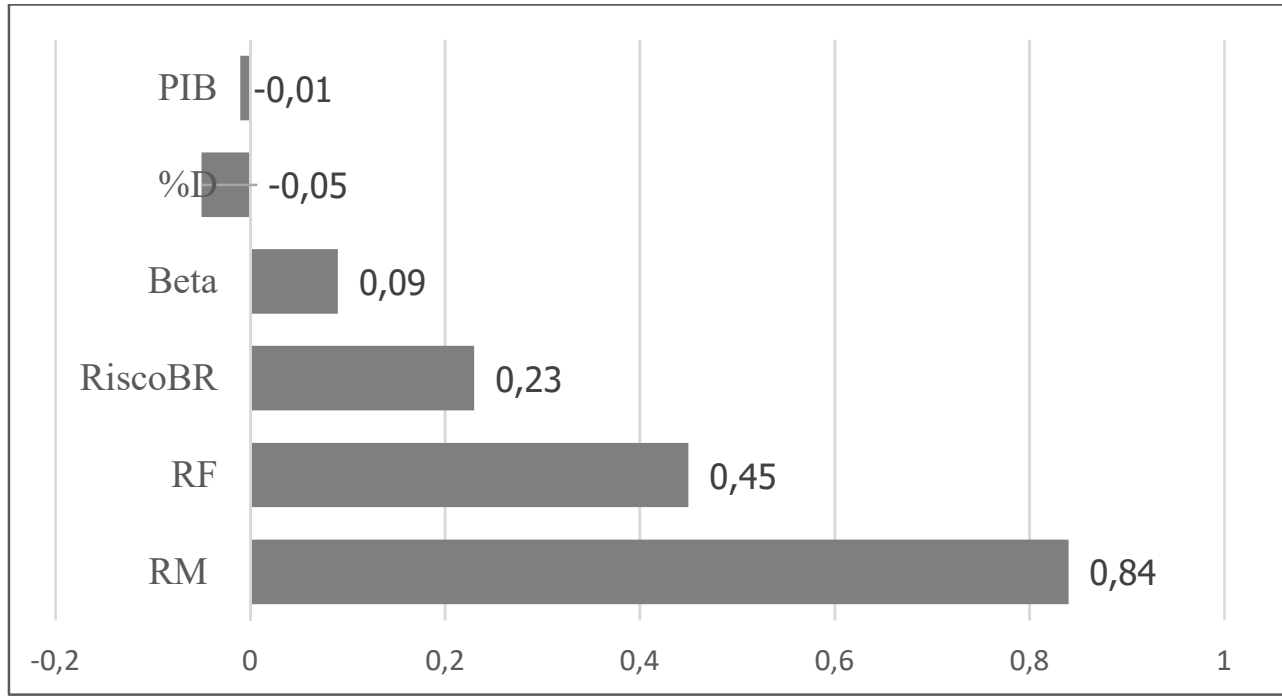

Figura 3: Correlações do WACC com as variáveis assumidas na simulação Fonte: Dados da pesquisa.

Analisando a Figura 3 é possível concluir que o retorno de mercado é responsável por $84 \%$ de variância no custo de capital. A segunda etapa de análise da simulação de Monte Carlo é verificar a simulação do valor de saída, que é o valor de mercado que a empresa Ambev pode assumir considerando as variações de custo de capital apresentadas. Após a simulação, foram gerados os dados estatísticos, de acordo com a Tabela 7.

Tabela 7: Dados estatísticos da simulação do Valor de Mercado

\begin{tabular}{lrlr}
\hline \multicolumn{4}{c}{ Valores de previsão } \\
\hline Avaliações & 10.000 & Curtose & 6,47596484 \\
Caso Base & $125.526,03$ & Coeficiente de Variação & 0,15299478 \\
Média & $139.572,08$ & Mínimo & $91.315,67$ \\
Mediana & $136.846,65$ & Máximo & $337.681,15$ \\
Desvio Padrão & $21.353,80$ & Largura do Intervalo & $246.365,48$ \\
Variância & 455.984 .788 & Erro Padrão Média & 213,538003 \\
Obliquidade & 1,11947437 & & \\
\hline
\end{tabular}

Fonte: Dados da Pesquisa

Com base na Tabela 7, considerando um intervalo de confiança de $95 \%$, os valores que a empresa Ambev pode assumir estão entre R\$ 91,315 bilhões, considerando o pior cenário possível e R \$337,681 bilhões, considerado o melhor cenário possível, assumindo o menor custo de capital. A média dos valores encontrados na simulação ficou em $\mathrm{R} \$ 139,572$ bilhões, esse 
valor está $11,18 \%$ acima do valor de mercado que foi encontrado no cálculo determinístico. As distribuições dos valores encontrados podem ser analisadas a partir da Figura 4.

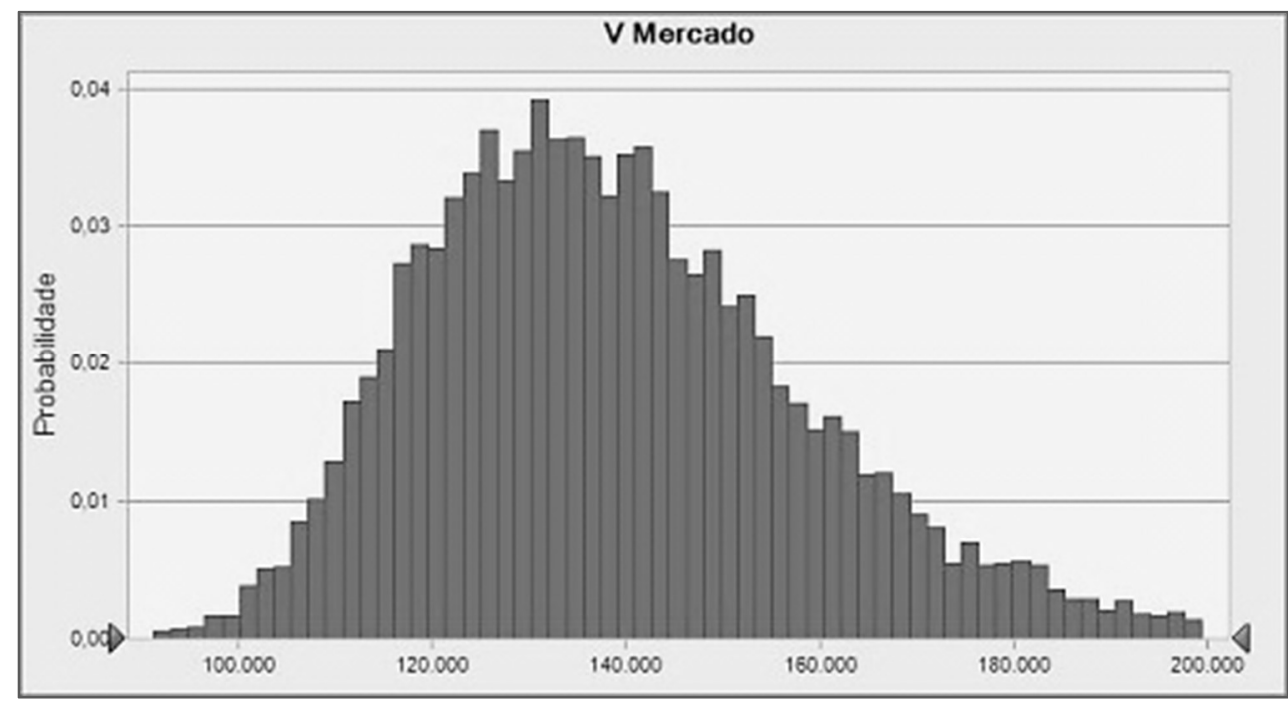

Figura 4: Distribuição de probabilidade do Valor de Mercado Fonte: Dados da pesquisa.

Assim como no WAAC, foi analisada a sensibilidade das variáveis no resultado final do valor de mercado. As variáveis que possuem uma relação negativa com o valor de mercado é o RM e o RF, quanto maior forem seus valores, menor será o valor da empresa. Enquanto o PIB estabelece uma relação positiva, quanto maior for o PIB, maior será o valor da empresa. A Figura 5 apresenta as correlações das variáveis do valor de mercado.

Revista ENIAC Pesquisa, Guarulhos (SP), V.8, n.1, jan.- jun. 2019. 


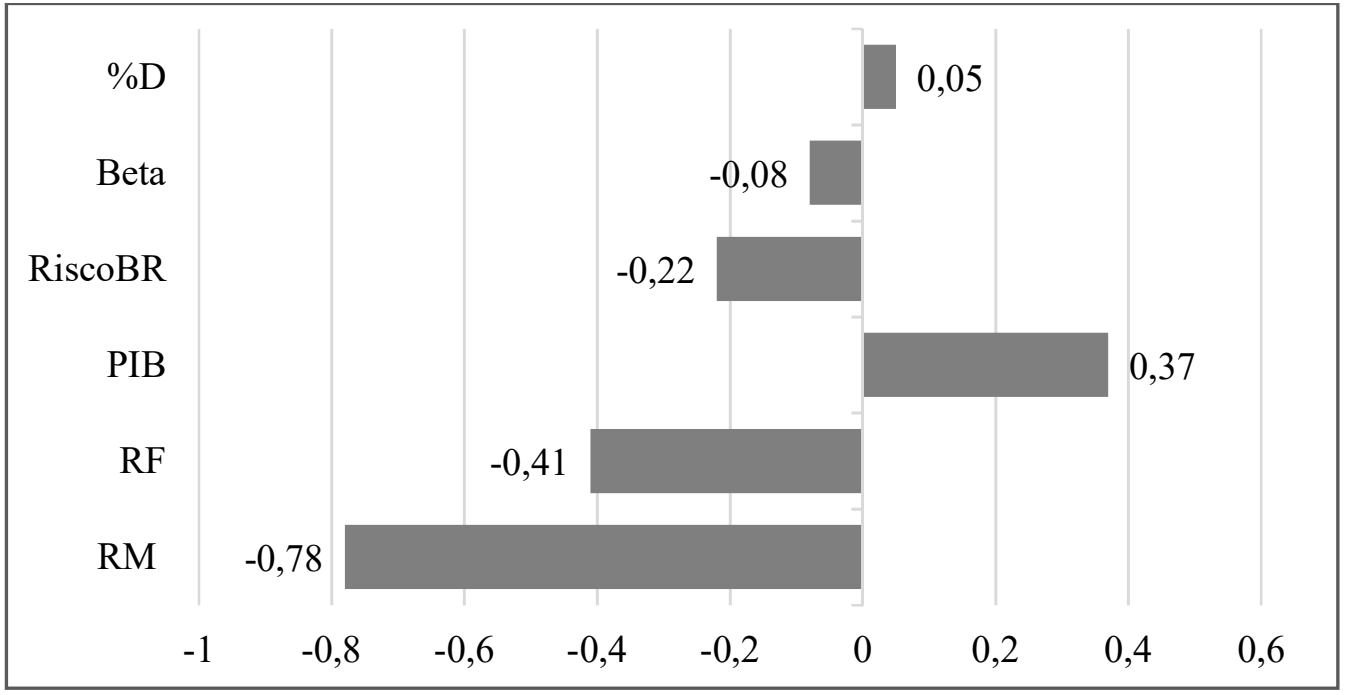

Figura 5: Correlações do valor de mercado com as variáveis assumidas na simulação Fonte: Dados da pesquisa.

Observa-se que todas as variáveis que formam o custo médio ponderado de capital WACC, com exceção do percentual de dívidas (Estrutura de capital) tem um relacionamento inverso com o valor de mercado, levando a entender que, quanto menores, maior será esse valor. Vê-se que a estrutura de capital, nos limites das variáveis apresentadas pela Ambev, poderia influenciar positivamente o valor de mercado, que se beneficiaria da alavancagem financeira. Entretanto, como exposto, a composição de dívidas na estrutura dessa empresa é bastante reduzida. O beta, coeficiente que mostra o perfil de risco da empresa com relação a volatilidade do mercado, seria um forte determinante do valor de mercado caso se tratasse de uma empresa com perfil "agressivo". Entretanto, a empresa Ambev é de baixo risco e a volatilidade dos seus retornos é mais condicionada ao desempenho da economia como um todo.

\section{CONSIDERAÇÕES FINAIS}

Este trabalho teve como objetivo verificar a acurácia da utilização da simulação de Monte Carlo no processo de avaliação de uma empresa através do método de fluxo de caixa descontado. A ênfase da simulação foi dada no cálculo da taxa de desconto utilizada no processo de avaliação, com o intuito de mitigar as incertezas que possui no contexto de um país emergente.

Revista ENIAC Pesquisa, Guarulhos (SP), V.8, n.1, jan.- jun. 2019. 
O método de Monte Carlo ajudou a entender quais os valores o WACC pode assumir em diversos cenários possíveis, e consequentemente o Valuation da empresa Ambev. E por isso, a simulação de Monte Carlo se mostra importante, pois através da sua utilização é possível entender a distribuição do valor que a empresa pode ter, bem como a probabilidade de sua ocorrência, o que permite compreender as incertezas ligadas às variáveis e suas relações, através da análise de sensibilidade realizada.

Neste trabalho, o valor encontrado para a empresa está dentro do intervalo de confiança da simulação de Monte Carlo, e o Valuation encontrado nos cálculos determinísticos, está próximo da média que foi encontrada nas simulações. Ressalta-se que mesmo o método de Monte Carlo pode trazer viés na precificação de um ativo, uma vez que a escolha das premissas que serão utilizadas para fazer a simulação, não está isenta da interpretação do analista da aplicação do método, o que leva a aumentar a possibilidade de encontrar diferentes resultados de Valuation. Ou seja, mesmo essa arbitragem e subjetividade seja menor no método determinístico, ainda assim pode ocorrer variações nos resultados de Valuation realizados por diferentes analistas. Contudo, apesar dessas limitações a simulação de Monte Carlo é uma importante ferramenta no que tange a análise de risco e tomada de decisão em ambientes de incerteza.

Diante do exposto, faz se as seguintes recomendações para trabalhos futuros: realizações de estudos com empresas com diferentes tipos de volatilidade e utilização de critérios quantitativos em todas as premissas das variáveis que forem simuladas.

\section{REFERÊNCIAS}

AMBEV. Ratings. On-line, 2018. Disponível em: $<$ http://ri.ambev.com.br/conteudo pt.asp?idioma $=0 \&$ tipo $=43221 \&$ conta $=28>$ Acesso em: 19 de maio de 2018.

AMSLER, E.; SCHMIDT, P. A Monte Carlo investigation of the accuracy of multivariate CAPM tests. Journal of Financial Economics, v. 14, n. 3, p. 359-375, 1985.

ARAUJO, A. M. P; LIMA, F. G.; ASSAF NETO, A. Metodologia de Cálculo do Custo de Capital no Brasil. In: Congresso Brasileiro de Contabilidade da USP. 2006.

ASSAF NETO, A. Finanças corporativas e valor. 3. ed. São Paulo: Atlas, 2010.

BRUNI, A. L. Avaliação de Investimentos. 2. ed. São Paulo: Atlas, 2013.

Revista ENIAC Pesquisa, Guarulhos (SP), V.8, n.1, jan.- jun. 2019. 
CARDOSO, D; AMARAL, H, F. O uso da simulação de monte carlo na elaboração do fluxo de caixa empresarial: Uma proposta para quantificação das incertezas ambientais. Encontro Nacional de Engenharia de Produção-ENEGEP, 2000.

CORREIA NETO, J, F; DE MOURA, H, J; FORTE, S, H, A, C. Modelo prático de previsão de fluxo de caixa operacional para empresas comerciais considerando os efeitos do risco, através do método de Monte Carlo. Revista Eletrônica de Administração, v. 8, n. 3, 2002.

DAMODARAN, A. Applied Corporate Finance 4. ed. New York: Wiley, 2014.

DAMODARAN, A. Finanças Corporativas: teoria e prática. 2. ed. Porto Alegre: Bookman. 2004.

DAMODARAN, A. Avaliação de investimentos. Rio de Janeiro: Qualitymark, 1997.

DAMODARAN, A. Annual Returns on Stock, T.Bonds and T.Bills: 1928 - Current. Online, 2018a. Disponível em: $<$ http://pages.stern.nyu.edu/ adamodar/New_Home_Page/datafile/histretSP.html $>$. Acesso em 19 de maio de 2018.

DAMODARAN, A. Ratings, Interest Coverage Ratios and Default Spread. On-line 2018b. Disponível em: http://pages.stern.nyu.edu/ adamodar/New_Home_Page/datafile/ratings.htm>. Acesso em 19 de maio de 2018.

DAMODARAN, A. Country Default Spreads and Risk Premiums. On-line 2018c. Disponível

$<$ http://pages.stern.nyu.edu/ adamodar/New_Home_Page/datafile/ctryprem.html $>$. Acesso em 19 de maio de 2018.

ECONOMATICA ${ }^{\circ}$. Petrobras retoma a liderança na América Latina por valor de mercado. Ação ordinária valoriza mais de $70 \%$ em 2018 até 10 de maio. Entre as grandes petroleiras é a oitava e sobe duas posições desde dezembro de 2017. On-line, 2018. Disponível em: $<$ http://economatica.com/estudos/data/20180511a.pdf $>$. Acesso em: 20 jul 2018.

FRENCH, N; GABRIELLI, L. Discounted cash flow: accounting for uncertainty. Journal of Property Investment \& Finance, v. 23, n. 1, p. 75-89, 2005.

FOCUS. Relatório de Mercado. Banco Central. On-line, 2018 Disponível em: $<$ https://www.bcb.gov.br/pec/GCI/PORT/readout/readout.asp.> Acesso em: 22 de fevereiro de 2018 .

GARRAN, F. T. Metodologias em uso no Brasil para a determinação do custo de capital próprio para avaliação de ativos por fluxo de caixa descontado. Tese de Doutorado. Universidade de São Paulo, São Paulo: 2006.

GIL, A. C. Como elaborar projetos de pesquisa. 4. ed. - São Paulo: Atlas, 2002. 
MASSARI, M; GIANFRATE, G; ZANETTI, L. Corporate valuation: Measuring the value of companies in turbulent times. John Wiley \& Sons, 2016.

MEDEIROS NETO, L. B. de. Análise de risco na avaliação econômico financeira de empresas: uma abordagem estocástica utilizando simulação de Monte Carlo. Recife, UFPE, 2009. 97 f. Dissertação (Mestrado em economia) - Universidade Federal de Pernambuco, Recife, 2009.

MÜLLER, A. N.; TELÓ, A. R. Modelos de avaliação de empresas. Revista da FAE, v. 6, n. 2, 2003.

OLIVEIRA, D. B.; BORGES, G. F; CUNHA, M. F.; QUEIROZ L. M. Petrobras: Um Caso Sobre O Valuation Para A Decisão De Investimentos Em Ações. Revista de Gestão, Finanças e Contabilidade, v. 7, n. 3, p. 397-413, 2017.

OLIVEIRA, M. R. G.; MEDEIROS NETO, L. B. M. Simulação Monte Carlo e Valuation: uma abordagem estocástica. REGE. São Paulo, v. 19, n. 3, p. 449-466, 2012.

SANTOS, T, B.; CUNHA, M. F. Avaliação de empresas: uma análise sob a ótica do "viés de avaliação" nos laudos de oferta pública de aquisição ações. Revista de Gestão, Finanças e Contabilidade, v. 5, n. 3, p. 61-74, 2015.

SCHNORRENBERGER, D.; AMBROS, M. G.; GASPARETTO, V. LUNKES, R. J. Comparação entre métodos para avaliação de empresas. Navus - Revista de Gestão e Tecnologia, v. 5, n. 1, p. 79-92, 2015.

SOUTE, D. O.; SCHVIRCK, E.; MARTINS, E.; MACHADO, M. R. C. Métodos de avaliação utilizados pelos profissionais de investimento. Revista UnB Contábil, v. 11, n. 1-2, p.1-17, 2008 .

TIBOR, T; VERONIKA, F; REKA, T. Corporate valuation using two-dimensional monte carlo simulation. Annals of the University of Oradea: Economic Science, v. 1, n. 2, p. 788-794, 2010 .

Revista ENIAC Pesquisa, Guarulhos (SP), V.8, n.1, jan.- jun. 2019. 